\title{
Editorial
}

\section{Nanoporous and Nanostructured Materials for Catalysis, Sensor, and Gas Separation Applications}

\author{
Songwei Lu \\ Glass Technology Center, PPG Industries, Inc., Pittsburgh, PA 15238-0472, USA
}

Received 31 December 2006; Accepted 31 December 2006

Copyright () 2006 Songwei Lu. This is an open access article distributed under the Creative Commons Attribution License, which permits unrestricted use, distribution, and reproduction in any medium, provided the original work is properly cited.

Nanostructures in the form of thin films, nanoparticles, nanoporous materials, nanocomposites, and bulk nanocrystalline materials are of interest both for basic scientific research and technological applications as their properties are dominated by the extremely large specific surface areas. Such surfaces have generally unique properties which greatly differ from those of bulk materials, and which may even acquire characteristic size dependence at the nanometer scale. Because of the high surface-to-volume ratio, local phenomena, such as adsorption or changes in the surface electronic state, may contribute significantly to the overall properties of the materials. The detailed understanding of nanoporous materials and nanostructured materials defines challenges in basic science, not only in synthesis but also with respect to characterization and modeling, for instance, of surfacerelated properties. In addition, these nanomaterials have an immense potential for technological applications in chemical production, environmental control, photovoltaic and energy systems.

This special issue of Journal of Nanomaterials titled "Nanoporous and nanostructured materials for catalysis, sensor, and gas separation applications" is based in part on papers submitted to the Materials Research Society (MRS) 2005 Spring Meeting, Symposium R "Nanoporous and nanostructured materials for catalysis, sensor, and gas separation applications," organized by Songwei Lu of PPG Industries, Inc., Horst Hahn of Technical University of Darmstadt, James Gole of Georgia Institute of Technology, and Jörg Weissmüller of Research Center Karlsruhe. The symposium was financially supported by the donors of the American Chemical Society Petroleum Research Fund and PPG Industries, Inc.

This special issue with a total of fifteen papers covers a wide range of topics related to nanoporous and nanostructured materials for catalysis and sensor applications, ranging from basic to applied materials research and focusing on synthesis, functionalization, processing, characterization, and applications. Among fifteen papers, nine papers were presented in the Symposium R during the MRS 2005 Spring Meeting, and six papers are from open call for papers for the special issue. It divides into four sessions: nanostructured materials for catalysis and photocatalysis applications; nanoporous materials; nanostructured materials for sensor applications; and nanomaterials synthesis and other applications.

The first session includes five papers focusing on nanostructured materials for catalysis and photocatalysis applications. The first paper "Ni hollow nanospheres: preparation and catalytic activity" by Kaifu Zhong et al. demonstrates a method to prepare monodispersed silica nanospheres as templates for fabrication of nickel-silica composite hollow spheres, which display relative high activity and selectivity in acetone hydrogenation, showing potential applications as nanocatalysts. In "Fractal dimension of active-site models of zeolite catalysts," F. Torrens and G. Castellano. calculated the active-site of zeolite catalysts and compared other calculation methods. While most of research focused on photocatalysis in UV region, Sesha S. Srinivasan et al. in their two papers explored the visible-light photocatalytic activities of $\mathrm{TiO}_{2}-\mathrm{ZnFe}_{2} \mathrm{O}_{4}$ nanoparticles and $\mathrm{CdS}-\mathrm{TiO}_{2}$ nanocomposite materials. Huifang $\mathrm{Xu}$ et al. described synthesis and photocatalytic property of titania nanotubes in their paper "Photocatalytic oxidation of acetaldehyde using titanium oxide nanotubes."

The second session with a topic of "nanoporous materials" includes four papers. Helmut Föll et al. in their paper "Porous and nanoporous semiconductors and emerging applications" briefly review pore formation, properties of porous semiconductors and emerging applications. The paper "Atomic layer deposition for the conformal coating of 
nanoporous materials" by Jeffrey W. Elam et al. used atomic layer deposition (ALD) to apply precise, conformal coatings over nanoporous anodic aluminum oxide (AAO) membranes and silica aerogels, which may have a great promise as hydrogen sensors. In "Size-dependent specific surface area of nanoporous film assembled by core-shell iron nanoclusters," Jiji Antony et al. presented synthesis and characterization of stable monodispersed iron-iron oxide core-shell nanoporous films, which may have applications in remediation and environmental treatments using their large surface area. In addition, T. T. Zhuang et al. described the capability of capturing volatile nitrosamines in airstreams using various modified zeolite in their paper titled "New development in nanoporous composites: novel functional materials for capturing nitrosamines in airstreams."

The third session including three papers is "nanostructured materials for sensor applications." Florian Voigts et al. reported a potential high-temperature oxygen sensor material using undoped and 1 at \% $\mathrm{Nb}$-doped strontium titanate nanoparticle films in their paper titled "Synthesis and characterization of strontium titanate nanoparticles as potential high-temperature oxygen sensor material." The paper titled "Ferromagnetic nanostructures incorporated in quasi-one-dimensional porous silicon channels suitable for magnetic sensor applications" by Petra Granitzer et al. describes a novel fabrication method for porous silicon/Ninanocomposite system which exhibits a twofold switching behavior of the magnetization curve at two different field ranges, indicating a possible application as a high magnetic field sensor based on a silicon technology. In "Gas sensors based on tin oxide nanoparticles synthesized from a mini-arc plasma source," Ganhua Lu et al. reported on the fabrication and characterization of a functional tin oxide nanoparticle gas sensor, exhibiting a fast response and a good sensitivity.

The last session "nanomaterials synthesis and other applications" includes three papers: "Effect of pressure on synthesis of Pr-doped zirconia powders produced by microwavedriven hydrothermal reaction" by Agnieszka Opalinska et al. describing a microwave hydrothermal synthesis of Prdoped zirconia nanocrystals; "Epoxy-carbazole polymeric network nanolayers for organic light-emitting devices" by Jin-Woo Park et al. regarding organic light-emitting devices; and "Incorporation of vanadium oxide in silica nanofiber mats via electrospinning and sol-gel synthesis" by J. E. Panels and Y. L. Joo outlining a unique synthesis method for vanadium oxide-silica nanofibers. 

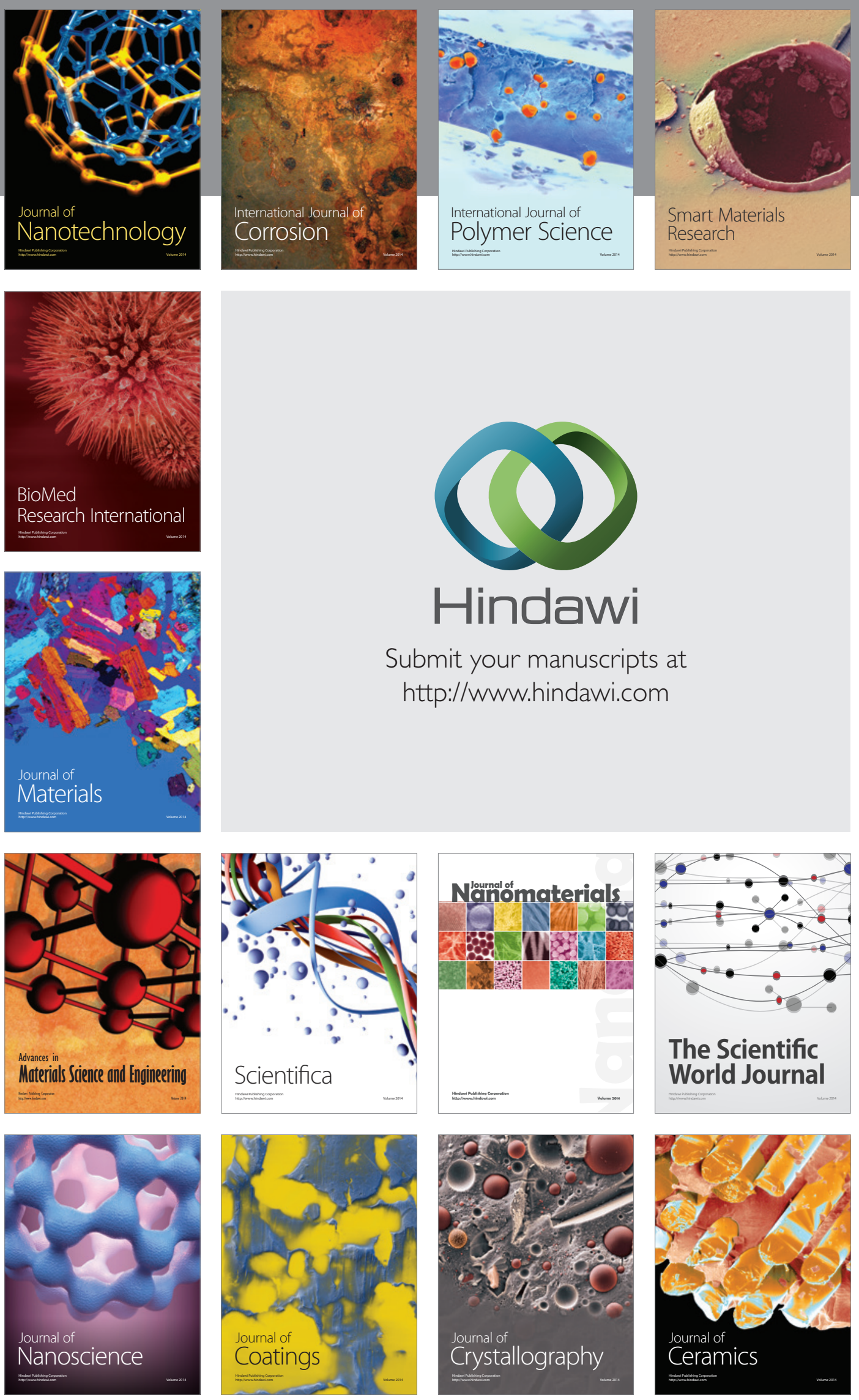

The Scientific World Journal

Submit your manuscripts at

http://www.hindawi.com

\section{World Journal}

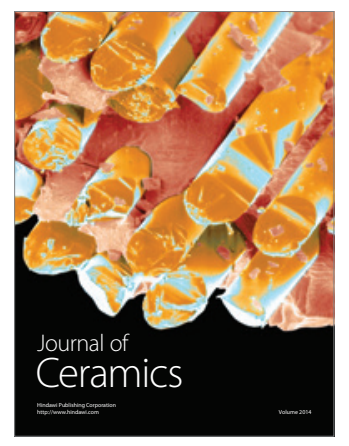

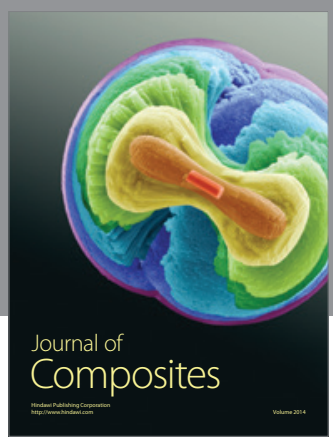
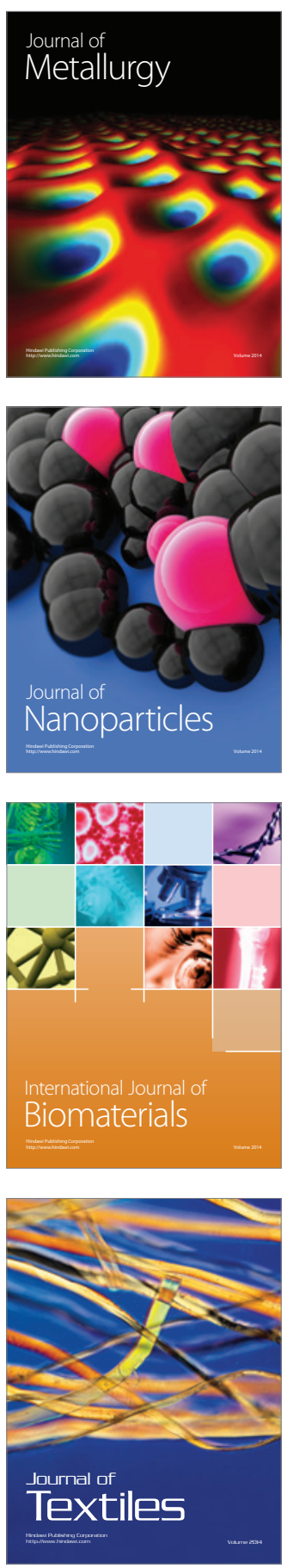